\title{
Large-Scale Molecular Dynamics Simulation of the Dehydration of a Suspension of Smectite Clay Nanoparticles: Supporting Information
}

\author{
Thomas R. Underwood* and lan C. Bourg* \\ Department of Civil and Environmental Engineering and Princeton Environmental \\ Institute, Princeton University, Princeton NJ 08544, United States
}

E-mail: thomas.underwood@princeton.edu; bourg@princeton.edu 


\section{Methodology}

A brief description of the molecular dynamics (MD) methodology and data analysis is presented in the article. This description is reproduced in full below.

\section{Model Setup}

\section{Setup of periodic clay sheets}

The clay examined in this study is a swelling Na-montmorillonite (Swy-2) with the stoichiometry: $\mathrm{Na}_{0.8}^{+}\left[\mathrm{Al}_{3.2} \mathrm{Mg}_{0.8}\right] \mathrm{Si}_{8} \mathrm{O}_{20}(\mathrm{OH})_{4}$. Initial structures of infinitely periodic clay sheets were generated via an $8 \times 5 \times 1$ expansion of the unit cell structure of pyrophyllite-1Tc (as per Lee and Guggenheim). ${ }^{1}$ This generated a sheet of pyrophyllite with an approximate geometry of $42 \AA \times 45 \AA \times 10 \AA$. Isomorphic charge substitutions of $\mathrm{Al}^{3+}$ to $\mathrm{Mg}^{2+}$ were randomly placed within the octahedral layer of the clay sheet subject to Pauling's fourth rule, such that no two $\mathrm{Mg}^{2+}$ charge substitutions share polyhedral elements with one an-

other. ${ }^{2}$ The system was subsequently charge balanced by placing $\mathrm{Na}^{+}$ions $5 \AA$ directly above these charge substitutions. Water molecules were randomly placed above the sheet of clay to generate $n$-layer hydrates of Na-montmorillonite. The requisite amount of water added per unit cell is presented in Figure 1 for each hydration state, as per Holmboe \& Bourg and Teich-McGoldrick et al. ${ }^{3,4}$ Finally, the single sheet of $n$-layer Na-montmorillonite was sufficiently replicated in $z$ such that the MD simulation supercell was at least $40 \AA$ in the $z$-direction. In total, 6 separate periodic clay models were generated, corresponding to the 0-, 1-, 2-, 3- and 4-layer crystalline hydrates of Na-montmorillonite; additionally, a system with a larger $6 \mathrm{~nm}$ nanopore spacing between clay sheets was produced to represent a larger osmotically metastable nanopore (as presented Figure 1). 

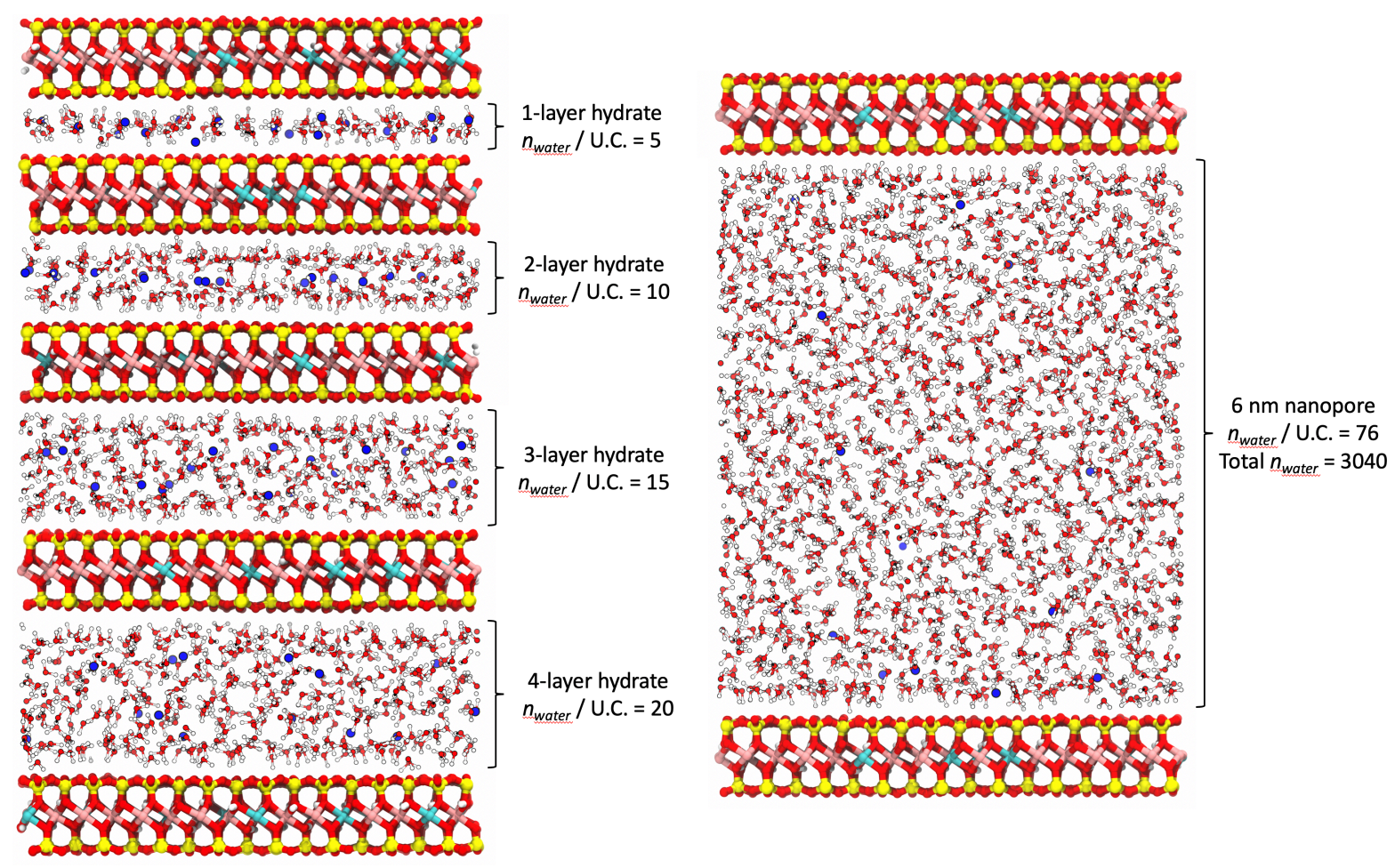

Figure 1: A schematic of the infinite lamellae simulations of Na-montmorillonite; crystalline hydrates are presented on the left, a $6 \mathrm{~nm}$ nanopore simulation is presented on the right.

\section{Setup of a single hexagonal clay particle}

The creation of hexagonal clay platelets follows a similar process to the creation of periodic sheets described above. Firstly, a large sheet of pyrophyllite was generated via a $24 \times 28$ $\times 1$ expansion of the pyrophyllite-1Tc unit cell. ${ }^{1}$ The generated slab of pyrophyllite was sufficiently large to create hexagonal clay platelets with an edge length of approximately 6 $\mathrm{nm}$, corresponding to a minor diameter of approximately $10 \mathrm{~nm}$, and a major diameter of approximately $12 \mathrm{~nm}$. The slab of pyrophyllite was cleaved into a hexagon along two [010] edges and four [110] edges. These edges were subsequently healed (i.e. hydrogen atoms were added to undercoordinated oxygen atoms) as per White and Zelazny at near neutral pH and will henceforth be referred to as the $\mathrm{B}$ and $\mathrm{AC}$ edges respectively. ${ }^{5}$ The final structure and a topological description of the montmorillonite edges are further elaborated upon in the following topological parameters section. Isomorphic charge substitutions of $\mathrm{Al}^{3+}$ to $\mathrm{Mg}^{2+}$ were randomly placed within the octahedral layer of the hexagonal particle, again, subject 
to Pauling's fourth rule. ${ }^{2}$ These charge substitutions were subsequently charge balanced by placing $\mathrm{Na}^{+}$ions $1 \mathrm{~nm}$ directly above/below the charge substitution. An example clay particle generated using this procedure is presented in Figure 2.
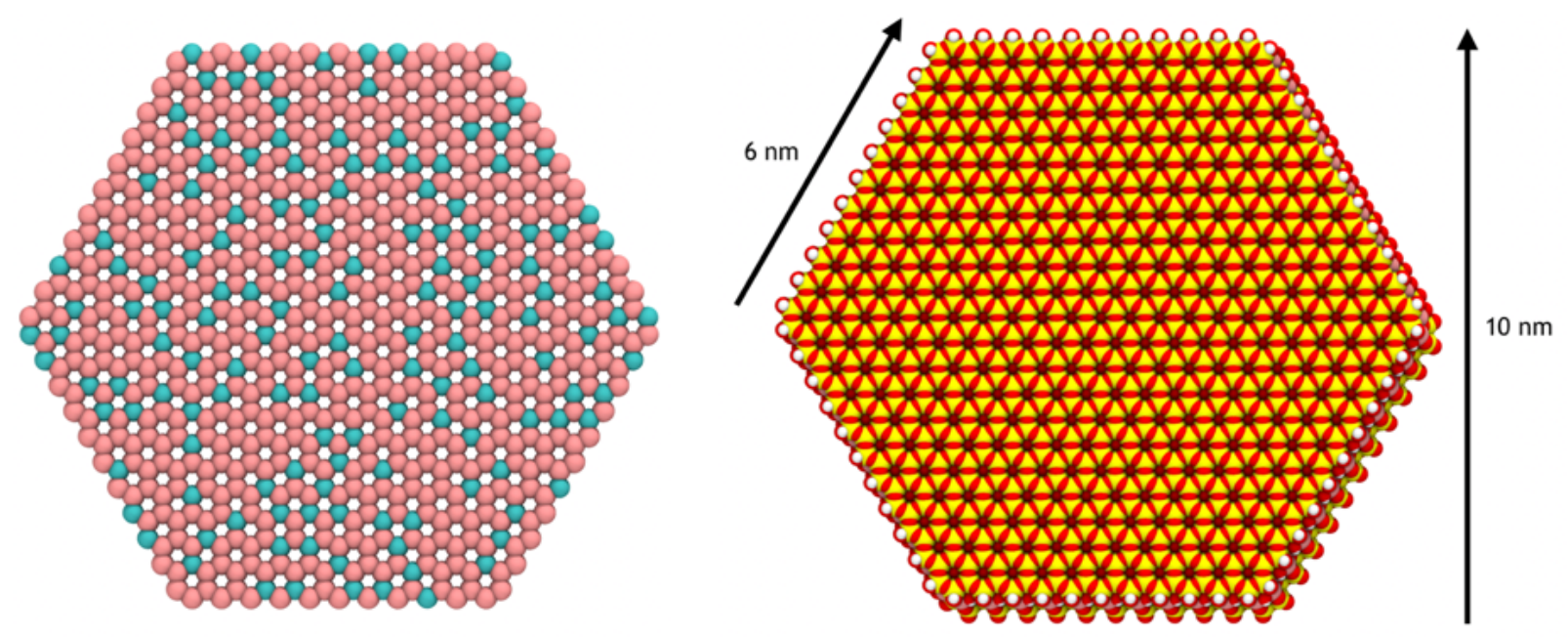

Figure 2: (Left) Example placement of isomorphic $\mathrm{Al}^{3+}$ to $\mathrm{Mg}^{2+}$ charge substitutions within the octahedral layer of the smectite clay particle. (Right) Example of the final clay particle $\left(\mathrm{Na}^{+}\right.$ions not shown).

\section{Topological Parameters}

The simulations examined in this study utilize the ClayFF force field to describe the interactions between all atoms within the Na-montmorillonite. ${ }^{6}$ The ClayFF force field is designed such that the majority of interactions within, and therefore the structure of, the clay is described wholly by the nonbonded Lennard-Jones and Coulomb potentials. The exception to this is the bonding of hydroxyl groups within the clay mineral, as well as optional angle terms between metal-oxygen-hydrogen groups. In our MD simulations of periodic montmorillonite sheets, all atoms are topologically typed as per their ClayFF counterparts. In addition, we utilize the SPC/E parameterization for water molecules as well as the Smith-Dang parameterization for $\mathrm{Na}^{+}$ions.

To model the edges of Na-montmorillonite we follow the procedure introduced by Lammers et al. ${ }^{7}$ Briefly, the structure of an edge is generated as described in the setup of a single 
hexagonal clay particle section. The Lennard-Jones parameters for edge atoms are assigned identical to their original ClayFF counterparts. It is important to note that upon healing the edges, we only add hydrogen atoms to undercoordinated oxygen atoms. Consequently, we set the partial charges of all non-oxygen atoms along the clay edge identical to their original partial charges as described by ClayFF. Upon constraining the charges of all non-oxygen atoms, we recalculate the partial charges on oxygen atoms $\left(Z_{O}^{\text {Partial }}\right)$ using a bond valence model:

$$
Z_{O}^{\text {Partial }}=-2.00+\sum_{i} \frac{\left(Z_{i}-Z_{i}^{\text {Partial }}\right)}{C N_{i}}
$$

where $Z_{i}$ is the valence of the $i$ 'th neighboring atom, $Z_{i}^{\text {Partial }}$ is the ClayFF assigned partial charge of the $i^{\prime}$ th neighboring atom, and $C N_{i}$ is the coordination number of the $i$ 'th neighboring atom. The summation in Equation 1 sums over only the nearest covalently bonded neighbors of the oxygen atom. This equation simply states that the charge on any oxygen atom is due to its inherent valence (-2 $q_{e}$, the first term in Equation 1), plus a contribution of shared positive partial charge from its nearest neighbors (the second term in Equation 1). It is also notable that this calculation is fully compatible with the original parameterization of ClayFF, i.e. this procedure can be utilized to calculate the partial charge of all oxygen atoms within the clay sheet (not just on the edges). A list of valences, ClayFF partial charges, and coordination numbers used in this calculation for various edge oxygen atoms is presented in Table 1.

Table 1: The bond valence parameters used in equation 1 to calculate the partial charge on edge site oxygen atoms. NB: In order to correctly model the edges of montmorillonite clay particles we slightly adjust the partial charges on octahedral magnesium substitutions (from $1.3600 \mathrm{q}$ to $1.3610 \mathrm{q}$ ), as well as the concomitant surrounding oxygen atoms (from $-1.0808 \mathrm{q}$ to $-1.0810 \mathrm{q}$ for adjacent hydroxyl oxygens, and from $-1.1808 q$ to $-\mathbf{1 . 1 8 1 0} q$ for adjacent bridging oxygens).

\begin{tabular}{l||ccc} 
Atom & $Z_{i}$ & $Z_{i}^{\text {Partial }}$ & $C N_{i}$ \\
\hline \hline Hydrogen & 1 & 0.425 & 1 \\
Silicon & 4 & 2.100 & 4 \\
Aluminum & 3 & 1.575 & 6 \\
Magnesium & 2 & 1.361 & 6
\end{tabular}


Using this method on the $\mathrm{AC}$ edge of Na-Montmorillonite at neutral $\mathrm{pH}$ produces the structure and partial charges presented in the top left schematic of Figure 3a. Using this parameterization, Lammers et al. discerned that the $\mathrm{OH}_{2}$ groups bonded to the octahedral metal atom of the clay would frequently ligand exchange with neutral water molecules. Furthermore, ab-initio DFT calculations further presented that the partial charges of the $\mathrm{OH}_{2}$ group is very similar to that of a water molecule, that there is little localised electron density at the midpoint of the $\equiv \mathrm{Al}-\mathrm{OH}_{2}$ bond, and that these $\mathrm{OH}_{2}$ groups would frequently detach from the edge of the clay and be replaced with a water molecule. ${ }^{7,8}$ Consequently, we have removed $\mathrm{OH}_{2}$ groups from the edges of the clay, transferring any residual charge to the now-undercoordinated octahedral metal cation (aluminum or magnesium). This process is presented in Figure 3a. 

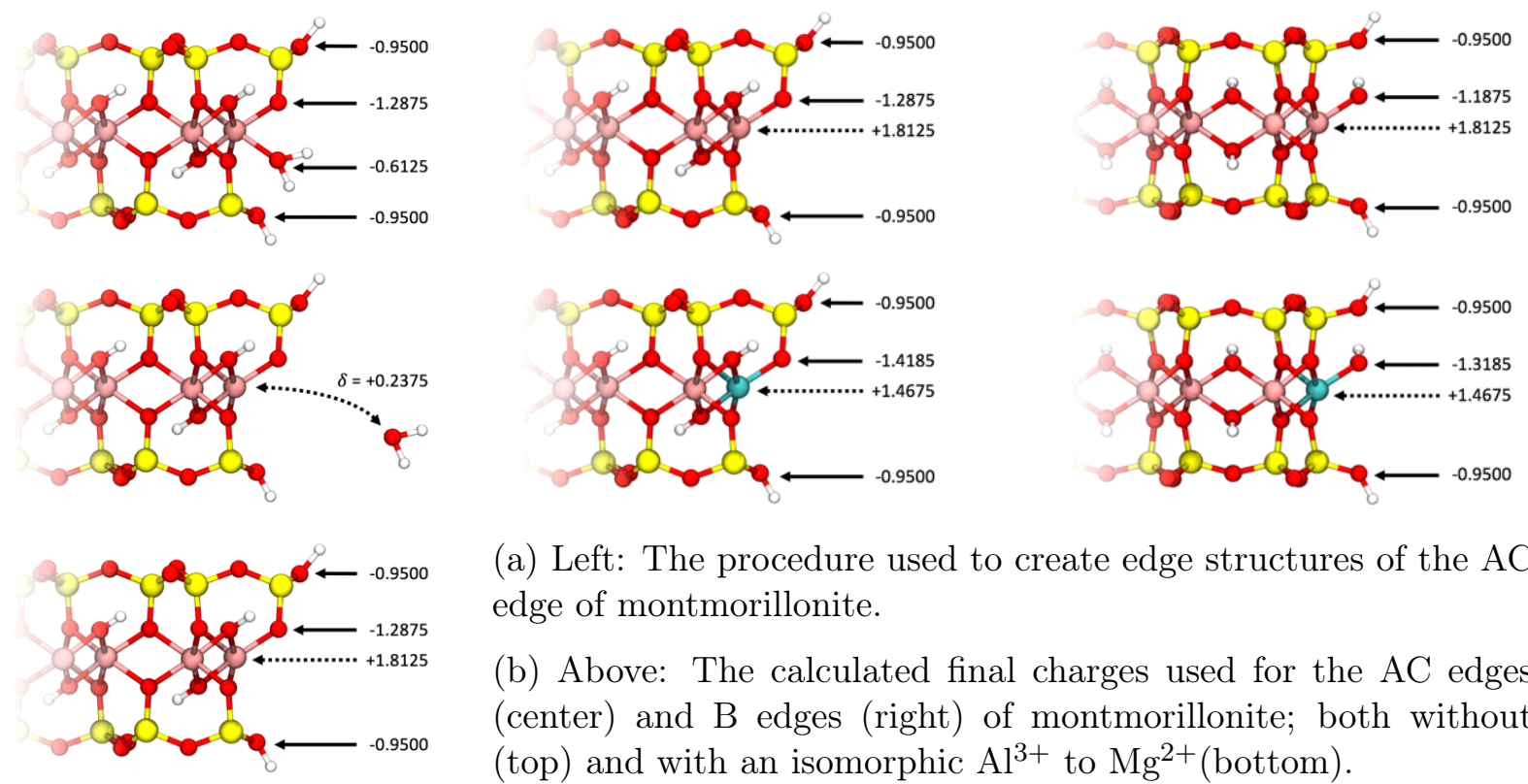

(a) Left: The procedure used to create edge structures of the AC edge of montmorillonite.

(b) Above: The calculated final charges used for the AC edges (center) and $\mathrm{B}$ edges (right) of montmorillonite; both without (top) and with an isomorphic $\mathrm{Al}^{3+}$ to $\mathrm{Mg}^{2+}$ (bottom).

Figure 3: The procedure used to create edge structures of the AC edge of montmorillonite. $\mathrm{OH}_{2}$ groups at the edge are converted to water molecules, and the residual charge is passed onto the underlying octahedral metal atom. Also presented is the final charges of edge oxygen and metal atoms for all permutations of edge sites within our model.

Using the simple procedure outlined above, i.e., healing the edge as per White and Zelazny at a given $\mathrm{pH}$; calculating the partial charge on edge oxygen atoms; and replacing $\mathrm{OH}_{2}$ groups with water molecules (subsequently transferring any change in charge from the $\mathrm{OH}_{2}$ groups to the underlying octahedral metal atom), the final structure and oxygen partial charges can be calculated for both the AC and B edge of Na-montmorillonite, both with and without isomorphic magnesium substitutions. These structures and partial charges are presented in Figure 3b. This process is also expected to work with other sorts of isomorphic substitutions (tetrahedral Al, for example), as well as with other verities of clay (2:1 illites and 1:1 kaolinites for example). Finally, we note that due to slight numerical instabilities observed over long-time scales along the AC edge of the clay, we implement metal-oxygenmetal harmonic potentials analogous to the study of Ho et al.. ${ }^{9}$ 


\section{Setup of bulk suspended clay systems}

To model bulk clay systems under compaction 30 discrete unique clay particles were initially randomly placed (both random in position and orientation) within a $24 \times 24 \times 40 \mathrm{~nm}^{3}$ box. The dimensions of the simulation cell were specifically chosen such that clay particles could hypothetically tessellate perpendicular to the $z$ axis, which would therefore minimize any potential bias for stacking defects. The hexagonal particles were then added sequentially such that no two clay particles overlap, until all 30 clay particles were successfully placed. Note that using this technique may lead to unintended orientational correlations between clay particles, especially if the requested packing density of clay is too large. To minimize this effect, 300 separate unique configurations of the 30 clay particles were generated. For each unique configuration we calculate a scalar order parameter $(S)$, which quantifies the amount of orientational correlations between clay particles. ${ }^{10}$ A completely isotropic sample of clay particles would have a $S=0$, whilst perfectly ordered clay particles would have a $S=1$. Liquid crystals, for example, typically have a $S$ in the range of 0.5 to 0.8 . $S$ is calculated firstly by deriving the director tensor of the system $\left(Q_{\alpha \beta}\right)$ as:

$$
Q_{\alpha \beta}=\frac{1}{N} \sum_{i=1}^{N}\left(\frac{3}{2} \hat{e}_{i \alpha} \hat{e}_{i \beta}-\frac{1}{2} \delta_{\alpha \beta}\right) \quad \alpha, \beta=x, y, z
$$

where $\hat{e}_{\alpha \beta}$ is the unit vector directed normal to the $i$ 'th clay's basal surface, $N$ is the amount of clay particles in the system and $\delta_{\alpha \beta}$ is the Kronecker delta function. Diagonalization of the director tensor returns three eigenvalues, the largest of which is the scalar order parameter $(S)$. The corresponding eigenvector for the $S$ component of the director tensor corresponds to the director of the system (i.e. the average orientation of the system). A histogram of the $S$ order parameters for the 100 unique initial configurations is presented in Figure 4 . We note that most of the generated configurations have slight, but not excessive, orientational ordering $(0.1<S<0.45)$, indicating that our initial packing of Na-montmorillonite is not overly excessive. From the 300 unique initial configurations we chose the most randomly 
oriented (the configuration with the lowest $S$ order parameter) as the starting configuration to examine the behavior of Na-montmorillonite under compaction. A snapshot of the starting configuration is presented in the original article (Figure 1).

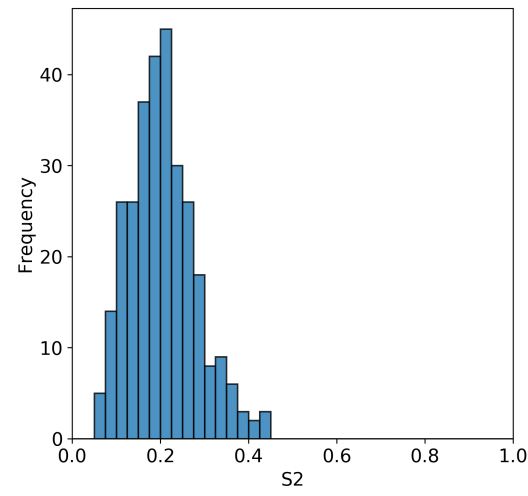

Figure 4: A histogram of $S$ parameters for the 300 separate initial configurations of 30 clay particles randomly placed within a $24 \times 24 \times 40 \mathrm{~nm}^{3}$ simulation box. The configuration with the lowest $S$ parameter was chosen as the initial seed for the compaction study.

The chosen system was subsequently solvated by tiling a pre-equilibrated box of SPC/E water within the $24 \times 24 \times 40 \mathrm{~nm}^{3}$ simulation. Water molecules overlapping (i.e. within 1.6 A) with a previously placed clay particle or sodium ion were deleted. Using this procedure, we generated an initial bulk clay system with randomly placed smectite clay particles (with minimal correlation between particles) at an initial water to clay mass ratio $(\mathrm{mW} / \mathrm{mC})$ of approximately 3. Overall, the final system contains 30 hexagonal Na-montmorillonite clay particles, each with a unique set of octahedral isomorphic substitutions and a major-diameter of $12 \mathrm{~nm}$, as well as 660,000 water molecules, amounting to over 2.4 million atoms in total. 


\section{Molecular Dynamic Simulations}

\section{Equilibration Simulations}

All molecular dynamics (MD) simulations have been performed using the GROMACS 2016.3

software package. ${ }^{11}$ All simulations were performed with periodic boundary conditions using a real space (van der Waals and electrostatic) cutoff of $12 \AA$, with long-ranged electrostatics calculated using the particle-particle-particle-mesh Ewald summation method with an accuracy of $99.95 \%$. All simulations were initialized with an energy minimization procedure to minimize any unphysical overlap between nearby atoms. This was accomplished using a steepest descents algorithm, with convergence accepted once the maximum force on any one atom was less than $1000 \mathrm{~kJ} \mathrm{~mol}^{-1} \mathrm{~nm}^{-1}$.

The minimized structures where then equilibrated for 100 ps in the NVT ensemble using a Berendsen thermostat with a time coupling constant of 1.0 ps at $298 \mathrm{~K}$ and an integration timestep of 1.0 fs. Initial particle velocities were assigned using a Boltzmann distribution at $298 \mathrm{~K}$. During the initial 50 ps of the NVT simulations, all non-hydrogen clay atoms and sodium ions were restrained using positional harmonic potentials. This allowed the surrounding water molecules to relax around the clay. During the subsequent 50 ps of the NVT equilibration, these restraints were removed.

Further equilibration simulations were then performed in the NPT and $\mathrm{NP}_{z} \mathrm{~T}$ ensembles using a Berendsen barostat with a pressure coupling constant of 1.0 ps at a pressure of $101.325 \mathrm{kPa}$. The NPT ensemble was used for systems containing periodic basal clay sheets, whilst the $\mathrm{NP}_{z} \mathrm{~T}$ ensemble was used for systems containing multiple discrete hexagonal clay particles. The temperature in the isothermal-isobaric ensemble simulations was maintained at $298 \mathrm{~K}$ using a velocity-rescale thermostat and a coupling constant of $1.0 \mathrm{ps}$ (as with the NVT equilibration simulations). Equilibration simulations in the isobaric-isothermal ensemble were run for $10 \mathrm{~ns}$.

Throughout all simulations, bonds involving a H-atom (within both clay and water) were 
restrained using the LINCS algorithm, whilst the geometry (bonds and angles) of each water molecule was further restrained using the SHAKE algorithm.

\section{Production Simulations - Procedure to recreate compacted clay systems}

Once the systems were equilibrated, production simulations in the isobaric-isothermal ensemble $\left(\mathrm{NPT} / \mathrm{NP}_{z} \mathrm{~T}\right)$ were calculated for both periodic basal clay and discrete hexagonal clay particle systems.

For the idealized systems of periodic Na-montmorillonite, production MD simulations in the NPT ensemble were carried out for 10 ns using a V-rescale thermostat at $298 \mathrm{~K}$ with a time-coupling constant of 1.0 ps and a Parrinello-Rahman barostat with a pressure coupling constant of $1.0 \mathrm{ps}$ at a pressure of $101.325 \mathrm{kPa}$. All analyses of these idealized systems were calculated over the final $5 \mathrm{~ns}$ of the $10 \mathrm{~ns}$ production simulation for each hydration state.

To reproduce the compaction of Na-montmorillonite, the system containing discrete hexagonal clay particles was simulated in the $\mathrm{NP}_{z} \mathrm{~T}$ ensemble using a V-rescale thermostat at $298 \mathrm{~K}$ with a time-coupling constant of 1.0 ps and a Parrinello-Rahman barostat with a pressure coupling constant of $1.0 \mathrm{ps}$ at a pressure of $101.325 \mathrm{kPa}$. After being simulated for $1 \mathrm{~ns}$, the simulation is paused and we randomly delete a select amount of water molecules from the system. The system is again simulated for $1 \mathrm{~ns}$, before more water is removed at a consistent rate. This process is repeated until all water molecules are removed from the system. This process is carried out sufficiently slowly to allow the clay nanoparticles to orient and form structures similar to those expected under real-world dehydration. Initially, every 1 ns we randomly remove 6,600 water molecules from the system (starting from an initial amount of approximately 660,000 water molecules). Once the system reaches a water to clay mass ratio of 1 , we slow down the dehydration rate such that we remove 3,300 water

molecules every $1 \mathrm{~ns}$. We observe that halving the dehydration rate at this step helps to reduce stacking defects in the simulation. Overall, the dehydration process takes over 134 ns to complete, excluding the additional 10 ns utilized to initially equilibrate the system. 
All analyses of the compacted system are calculated using the final $0.5 \mathrm{~ns}$ of each 1 ns dehydration step.

\section{Data Analyses}

\section{Calculation of Porosity}

One important feature of the microstructure of a clay matrix is porosity. In the present study, we calculate porosity using two separate techniques.

Firstly, as we know the exact number of water molecules in our simulation at every step during compaction, we calculate the volume that such an amount of water would occupy assuming it behaves like bulk liquid water at the corresponding temperature and pressure. The ratio of this expected volume against the overall system volume returns the volume fraction of water in the clay matrix throughout dehydration. Throughout the rest of the paper, we refer to this technique as the expected water volume method.

The second technique utilized to calculate the porosity of the clay matrix is the hardsphere test-particle insertion (TPI) method. In this technique, we randomly insert a hardsphere with a given radius into a simulated configuration of the clay matrix at each point during compaction. If the inserted sphere does not overlap with any clay atom then the attempt is recorded as successful. The calculated porosity $(\phi)$ is subsequently given as:

$$
\phi=\frac{N_{\text {successful }}}{N_{\text {total }}}
$$

where $N_{\text {succesful }}$ is the amount of successful insertions and $N_{\text {total }}$ is the total amount of attempted insertions. Note that in the large $N_{\text {total }}$ limit, this definition of porosity converges to the typical definition of porosity; i.e. the ratio of free- or pore-volume against total system volume. In this study we have tested various amounts of trial insertions. We have discerned that an $N_{\text {total }}$ above 1500 insertions attempts $\mathrm{nm}^{-3}$ returned porosities with a precision of greater than $0.05 \%$. Consequently, we insert hard-sphere test particles with a density of 1500 
insertions attempts $\mathrm{nm}^{-3}$ into the clay matrix at each stage of compaction.

Special consideration was taken in choosing the hard-sphere radii assigned to each atom belonging to the clay particles. Firstly, we assign the octahedral metal atoms (aluminum and magnesium) radii to $3 \AA$, in an attempt to block the inserted test-particles probing any internal cavities of the clay. The radii of all hydrogen and silicon atoms are assigned as 1.2 $\AA$ and $2.1 \AA$, as per their Van der Waals radii according to Bondi. ${ }^{12}$ Finally, we calculate the radius of oxygen atoms belonging to the clay using two independent methods. In the first method, we calculate the Gibbs dividing surface of water at the montmorillonite-water interface using the $6 \mathrm{~nm}$ nanopore model system. The Gibbs dividing surface describes the position of the water about the interface if we replaced the fluctuating density profile of water with a step function. We calculate that the Gibbs dividing surface of water is $1.56 \AA$ away from the apex of the apical basal oxygen atoms. In the second method, we fit a straight line to the porosity obtained using the expected water volume calculation between dry bulk densities of $0.3 \mathrm{~g} \mathrm{~cm}^{-3}$ to $0.6 \mathrm{~g} \mathrm{~cm}^{-3}$ (i.e. when the system contains large amounts of bulk-like water). This curve is subsequently extrapolated to zero water content, to obtain the ideal dry bulk density at zero water content $\left(\rho_{0}\right)$. Using this method, we obtain a hypothetical crystalline dry bulk density $\left(\rho_{0}\right)$ of $2.78 \mathrm{~g} \mathrm{~cm}^{-3}$, in-keeping with experimental observations. ${ }^{13}$

Using the unit cell structure of montmorillonite measured from $\mathrm{XRD}^{14}$ (i.e. using the $x$ and $y$ unit cell lengths measured from XRD, but not fixing the length in $z$ ), and the stoichiometry of the simulated Na-montmorillonite, we back-calculate a dehydrated $d$-spacing of $9.66 \AA$. From XRD, we also note that the apical basal oxygen atoms on either side of a clay sheet are $6.54 \AA$ apart. ${ }^{14}$ Subsequently, we would expect that at zero water content, the oxygen atoms would have a radius of $1.56 \AA$ as required to maintain a $d$-spacing of $9.66 \AA$ and a crystalline dry bulk density of $2.78 \mathrm{~g} \mathrm{~cm}^{-3}$. As validated by the two separate methods, we assign a hard-sphere radius of $1.56 \AA$ to all oxygen atoms in the clay. The overall porosity accessible to a water molecule $(\phi)$ is subsequently calculated using a probe sphere of radius $0.0 \AA$. 


\section{Pore-Size Distribution based on the distance to nearest surface $\left(P S D_{1}(r)\right)$}

We have further calculated the pore-size distribution (PSD) of the clay matrix at each stage of compaction using the techniques described by Gelb \& Gubbins, Sarkisov \& Harrison, and Torquato \& Avellenada. ${ }^{15-17}$ This definition of the PSD has dimensions of [length ${ }^{-1}$, and the value $P S D_{1}(r) d r$ can be interpreted as the probability that a randomly chosen point in the pore-space of the clay matrix lies a distance between $r$ and $r+d r$ from the pore-clay interface, where the pore-clay interface is equivalent to the Connolly surface of water about the clay particles, also known as the solvent accessible surface area. An alternate description of the $P S D_{1}(r)$ is that the quantity $P S D_{1}(r) d r$ describes the fraction of void space coverable by the centers of spheres of radius $r$, but not by the centers of spheres of radius $r+d r$. To calculate the pore-size distribution, we have carried out hard-sphere TPI calculations with particles of varying radii, from $0.0 \AA$ to $5.0 \AA$ in steps of $0.2 \AA$, from 5.0 to $10.0 \AA$ in steps of $1.0 \AA$, and from $10.0 \AA$ to $40.0 \AA$ in steps of $10.0 \AA$. We subsequently define the relationship between the pore-size distribution $\left(P S D_{1}\right)$ and the fraction of pore-space accessible to a hard-sphere probe of radius $r(F(r))$ as:

$$
\int_{r}^{\infty} P S D_{1}(R) \mathrm{d} R=F(r)
$$

where:

$$
\int_{0}^{\infty} P S D_{1}(R) \mathrm{d} R=1
$$

and consequently:

$$
\begin{gathered}
F(r=0)=1 \\
F(r=\infty)=0
\end{gathered}
$$


The fraction of pore-space accessible to the center of a hard-sphere probe of radius $r(F(r))$ has been calculated at each point during compaction as:

$$
F(r)=\frac{\phi(r)}{\phi(r=0)}
$$

where $\phi(r)$ is the volume accessible to a probe-sphere of radius $r$ calculated using TPI, and $\phi(r=0)$ represents the total porosity available to water molecules as described in the porosity calculation in the previous section.

Using the relationship in equation 4 we calculate the $P S D_{1}(r)$ of the clay matrix at each point during compaction as: ${ }^{15-17}$

$$
P S D_{1}(r)=-\frac{d F(r)}{d r}
$$

where the negative sign has been introduced upon flipping the limits of integration in equation 4 and applying the second fundamental theorem of calculus. The solvent-accessible solvent area (SASA), i.e. Connolly surface of water about the clay particles as, can subsequently be calculated as:

$$
S A S A=P S D_{1}(r=0)=-\lim _{r \rightarrow 0} \frac{d F(r)}{d r}
$$

Using these definitions, we can further quantify the accessible pore-fraction for the first hydration layer (1W) of water surrounding the clay minerals as:

$$
\int_{0}^{1 W} P S D(R) d R=\int_{0}^{\infty} P S D(R) d R-\int_{1 W}^{\infty} P S D(R) d R=F_{0}-F_{1 W}
$$

Similarly, the volume fraction available for the second and third hydration layers of water surrounding the clay is given as $\left(F_{1 W}-F_{2 W}\right)$ and $\left(F_{2 W}-F_{3 W}\right)$ respectively. These hydration layers represent surface coatings of water molecules around the clay particle, and are finite quantities for clays in dilute suspension (i.e. they do not necessarily quantify the amount of water within crystalline hydrates of the clay matrix). 
Finally, we note that the formation of one-layer crystalline hydrates corresponds to a system containing water within the first hydration layer of the clay $\operatorname{suspension}(i . e$. water located between $0 \AA$ and $1.5 \AA$ from the clay particles surfaces), with an absence of a corresponding second hydration layer (i.e. water located between $1.5 \AA$ and $3.0 \AA$ from the clay surfaces). Subsequently, we calculate the volume fraction consisting of one-layer hydrates as $F_{1-\text { layer }} \approx\left(F_{0}-F_{1 W}\right)-\left(F_{1 W}-F_{2 W}\right)$. Similarly, the volume fraction consisting of two-layer hydrates has been calculated as $F_{2-\text { layer }} \approx\left(F_{1 W}-F_{2 W}\right)-\left(F_{2 W}-F_{3 W}\right)$, and the volume fraction consisting of three-layer hydrates as $F_{3-\text { layer }} \approx\left(F_{2 W}-F_{3 W}\right)-\left(F_{3 W}-F_{4 W}\right)$. In all cases we assign radii of $r_{0}=0 \AA ; r_{1 W}=1.5 \AA ; r_{2 W}=3.0 \AA ; r_{3 W}=4.5 \AA$ and $r_{4 W}=$ $6.0 \AA$.

We note that the main approximation in the resulting volume fractions is that the calculations do not account for the effect of clay edges.

\section{Pore-Size Distribution based on the distance to the two nearest surfaces $\left(P S D_{2}(r)\right)$}

As an alternative method of calculation, we have calculated the pore-size distribution by calculating, for each water molecule, a pore-size metric $(r)$ :

$$
r=\frac{1}{2}\left(n_{\text {nearest-neighbor }}+n_{\text {second-nearest-neighbor }}\right)-r_{O B}
$$

where $n_{\text {nearest-neighbor }}$ and $n_{\text {second-nearest-neighbor }}$ are the distances from the water molecule of interest to its two nearest basal clay oxygen atoms on non-identical clay particles, and $r_{O B}$ is the radius of a basal clay oxygen atom, set to $r_{O B}=1.56 \AA$ as previously discussed. This pore-size metric, $r$, approximates the pore width of a channel accessible to a water molecule. The pore-size distribution for the clay mineral matrix $\left(P S D_{2}(r)\right)$ can subsequently be calculated by histogramming (and normalising) the distance metrics $(r)$ for all water molecules in the system. Compared to the calculation of $P S D_{1}(r)$, this technique has the disadvantage of being more challenging to interpret in pores with irregular shapes. For clearly defined pore 
separations, however, this metric is extremely useful. In the case of ideal periodic basal clay sheets, the pore-size metric $r$ displays clear cutoffs for model 1-, 2-, 3- and 4-layer hydrates. We subsequently identify 1-layer hydrate water molecules possessing $r<1.5 \AA$; 2-layer hydrate water molecules possessing $1.5 \AA \leq r<3.0 \AA$; and 3-layer hydrate water molecules as $3.0 \AA \leq r<4.5 \AA$. We ignore all water molecules with $r>4.5 \AA$, as 4-layer hydrates aren't expected to be present in large quantities for Na-montmorillonite. Using this calculation, we are able to identify the fraction of water molecules located in different types of crystalline hydrates. Furthermore, we can identify water molecules that are interfacial, but not contributing to crystalline hydrates (as ones with $n_{\text {nearest-neighbor }}<<n_{\text {second-nearest-neighbor }}$ ), and bulk-like water molecules (as $n_{\text {nearest-neighbor }}>>0$ and $n_{\text {second-nearest-neighbor }}>>0$ ).

\section{Enthalpy of Hydration}

Throughout the simulation, the enthalpy of the system has been recorded and is given as $H$. Initially, this is presented as a function of the water-clay mass ratio and is further manipulated to be directly comparable to calorimetric measurements. The enthalpy of the system at a given hydration state $\left(H\left(m_{W} / m_{C}\right)\right.$ in units of $\mathrm{kJ} / \mathrm{kg}_{c}$ (energy per mass of clay)) is given as:

$$
H\left(m_{W} / m_{C}\right)=H_{d r y}-\int_{0}^{m_{W} / m_{C}}\left(\hat{h}_{\text {ideal }}^{\text {vap }}+\hat{h}_{\text {excess }}^{\text {vap }}\right) d\left(m_{W} / m_{C}\right)
$$

where $H_{d r y}$ is the enthalpy of the system in the absence of any water, $\hat{h}_{\text {ideal }}^{\text {vap }}$ is the specific enthalpy of vaporisation of a water molecule from bulk water and $\hat{h}_{\text {excess }}^{\text {vap }}$ is the excess specific enthalpy of vaporisation of a water molecule due to the presence of the clay minerals. The specific partial enthalpies of vaporisation $\left(\hat{h}_{\text {ideal }}^{v a p}\right.$ and $\left.\hat{h}_{\text {excess }}^{\text {vap }}\right)$ can therefore be calculated from the enthalpic output of the simulation as:

$$
\frac{d H\left(m_{W} / m_{C}\right)}{d\left(m_{W} / m_{C}\right)}=\hat{h}_{\text {ideal }}^{v a p}+\hat{h}_{\text {excess }}^{\text {vap }}
$$


where $\hat{h}_{\text {ideal }}^{\text {vap }}$ is a known constant and $\hat{h}_{\text {excess }}^{\text {vap }}$ varies with clay content (or the water-clay mass ratio).

Finally, the enthalpy released going from a clay sample at a known hydration state, to a fully hydrated clay system, can be calculated and compared directly to results from immersion calorimetry as:

$$
\Delta Q_{\text {calorimetry }}=-\int_{m}^{\infty}\left(\frac{d H\left(m_{W} / m_{C}\right)}{d\left(m_{W} / m_{C}\right)}-\hat{h}_{\text {ideal }}^{\text {vap }}\right) d m_{\text {water }}=-\int_{m}^{\infty} \hat{h}_{\text {excess }}^{\text {vap }} d m_{\text {water }}
$$

\section{Activity of Water}

Throughout our simulation of the dehydration of Na-montmorillonite, water molecules are slowly removed at random. As an alternative approach, one could run a simulation using a Monte-Carlo (MC) algorithm (or a hybrid MC/MD algorithm) at a set chemical potential of water, by either slowly reducing the chemical potential to mimic the dehydration of the clay, or slowly increasing the vertical stress of the system to mimic the compaction of clay in a manner more akin to those experimentally resolved. Unfortunately, this approach is not currently possible, as such MC algorithms neither scale well with system size, nor parallelize sufficiently well over the required amount of computational resources necessary to complete an all-atom simulation of the clay matrix. With this noted, we have calculated the chemical potential/activity of water at each stage of dehydration using the Widom testparticle insertion technique. ${ }^{10,18}$ This technique is similar to the hard-sphere test-particle insertion described previously, however, instead of inserting a hard-sphere and checking for atomic overlap, an explicit water molecule is inserted at a random position and with a random orientation into the clay matrix and the non-bonded interaction energy $(\Delta U)$ between the inserted water molecule and the surrounding clay matrix is recorded. The excess chemical potential of water is then calculated as:

$$
\mu_{\text {excess }}=-k T \ln \left\langle\exp \left(\frac{-\Delta U}{k T}\right)\right\rangle=\mu-\mu_{\text {ideal }}
$$


where the angle brackets denote an average over many insertion attempts, $\mu$ is the total chemical potential of water in the system, and $\mu_{\text {ideal }}$ represents the chemical potential of an ideal gas at identical pressure, temperature and particle number. In the current study, we insert water molecules into the clay matrix at a density of 60,000 trial insertions $\mathrm{nm}^{-3}$. For each trial insertion location, a further 5000 trial insertions are attempted allowing the water molecule to rotate and translate within a sphere of $0.5 \AA$ about the molecule's center of mass. In total, our calculation of the excess chemical potential of water is calculated using 300 million trial insertions $\mathrm{nm}^{-3}$, meaning that at least 1 trillion trial insertions are calculated during each stage of dehydration (and more-so whilst the system is largely hydrated).

An additional complication to address in the calculation of the activity of water is due to the fact that the Widom insertion trial attempts are indiscriminately located within the clay matrix, whilst in reality, water molecules can only access the pore-spacing within the clay matrix (i.e. the method also attempts to insert water molecules within the structure of the clay). To account for this, we re-weight the excess chemical potential by the porosity of the clay matrix at each stage during compaction. Notably, the probability of an Widom insertion entering the pore-space (and avoiding a clay particle) is exactly $\phi$. This probability has a corresponding free energy $\left(\mu_{\phi}\right)$ given as:

$$
\mu_{\phi}=-k T \ln \phi
$$

and consequently, the chemical potential of water $(\mu)$ in the system is calculated as:

$$
\mu=\mu_{\text {ideal }}+\mu_{\text {excess }}-\mu_{\phi}
$$

The difference in the chemical potential of water and a reference state of bulk liquid water (at identical particle number, temperature and pressure) is given as:

$$
\Delta \mu=\mu-\mu^{b u l k}=\mu_{\text {excess }}-\mu_{\text {excess }}^{\text {bulk }}-\mu_{\phi}
$$


where $\mu_{\text {excess }}^{\text {bulk }}$ represents the excess chemical potential of bulk liquid water. Finally, the activity, or relativity humidity, of the system has be subsequently calculated as:

$$
a=\exp \left(\frac{\Delta \mu}{k T}\right)=\exp \left(\frac{\mu_{\text {excess }}-\mu_{\text {excess }}^{\text {bulk }}-\mu_{\phi}}{k T}\right)=\phi \exp \left(\frac{\mu_{\text {excess }}-\mu_{\text {bulk }}}{k T}\right)
$$

\section{Total Suction}

The total suction of water in the the simulation has been calculated using the conceptual system introduced by Sato (2008) and is consistent with the original developments of DLVO theory. ${ }^{19-21}$ In this methodology, the dehydrating clay matrix is chemically coupled to a bulk liquid phase separated by a semi-permeable membrane. During our dehydration process we conceptualise that the water molecules transfer from the clay matrix to the bulk liquid phase, rather than evaporating into a separate vapour phase. Consequently, we analyse this conceptual system in the canonical ensemble, using the following fundamental equation of thermodynamics:

$$
d F=-S d T-p d V+\sum_{i} \mu_{i} d n_{i}
$$

where $F$ is the Helmholtz free energy of the system, $S$ is the entropy of the system, $T$ the temperature, $p$ the pressure, $V$ the volume, $\mu_{i}$ is the chemical potential of species $i$, and $n_{i}$ is the amount of species $i$ contained within the larger compound system. We postulate that the total suction $\left(p_{\text {suction }}\right)$ can be calculated as:

$$
p_{\text {suction }}=-\left(\frac{\partial F}{\partial V}\right)_{T, n_{i}}=-\left(\frac{\partial F}{\partial n_{\text {water }}}\right)_{T, n_{j}}\left(\frac{\partial n_{\text {water }}}{\partial V}\right)_{T, n_{j}}=-\frac{\Delta \mu}{A_{x y}}\left(\frac{\partial n_{\text {water }}}{\partial z}\right)_{T, n_{j}}
$$

where $\Delta \mu$ is the chemical potential difference of water between the clay phase and the bulk liquid water reference state, $A_{x y}$ is the area of the simulation in the $x y$ plane, and the fraction $\left(\partial n_{\text {water }} / \partial z\right)$ describes the rate of change of water content with system size or dry bulk density. Notably, we observe that this derivation is similar to the expressions derived by Sposito ${ }^{22}$ and Sato; ${ }^{19}$ which state that the swelling pressure of a clay suspension 
in equilibrium with a bulk water reservoir at a constant temperature can be calculated as:

$$
\Delta p_{\text {swelling }}=-\frac{\Delta G_{H_{2} O}}{V_{w}}
$$

where $G_{\mathrm{H}_{2} \mathrm{O}}$ is the difference between the chemical potential of water in the clay suspension and that in a bulk water reference state, and $V_{w}$ is the specific volume of water.

We also note that our calculation of the total suction is calculated at each stage of compaction using configurations generated in the $N P_{z} T$ ensemble, whilst experimental results are generally calculated at a set relative humidity, i.e. the $\mu V T$ ensemble.

\section{Diffusion Coefficients of Water and Ions and their Associated Tortuosity}

The diffusion coefficients of both water molecules and sodium ions have been calculated using the well-accepted approach based upon the mean-squared displacement of each constituent component. ${ }^{10,23}$

The antisymmetric diffusion coefficient tensor of a species $\left(D_{i i}\right)$ is calculated as:

$$
D_{i i}=\lim _{\tau \rightarrow \infty} \frac{\left\langle\left(r\left(t_{0}+\tau\right)-r\left(t_{0}\right)\right)^{2}\right\rangle}{2 \tau}
$$

where $r$ is the position of the molecule in $\{x, y, z\}$ and the value $\left(r\left(t_{0}+\tau\right)-r\left(t_{0}\right)\right)$ represents the displacement of a single molecule between time $t_{0}$ and time $t_{0}+\tau$. The antisymmetric diffusion tensor possesses null values for off diagonal components $\left(D_{x y}=D_{x z}=0\right.$, etc.) and it is shown in the article that the diagonal components follow the expected trend: $D_{x x}=D_{y y} \neq D_{z z} \neq 0$.

The apparent tortuosity tensor of the system has also been calculated using the following relationship:

$$
D_{i i}=\tau_{i i} \sum_{n} D_{i i}^{n} f^{n}
$$

whereby $D_{i i}^{n}$ represents the diffusion coefficients of water or sodium in an $n$-layer hydrate of 
montmorillonite, and $f^{n}$ represents the fraction of all water or sodium ions forming $n$-layer hydrates during the dehydration of the system. The fraction of $n$-layer hydrates $\left(f^{n}\right)$ have been calculated using the distance-based metric introduced in the previous sections, and the diffusion coefficients in crystalline hydrates have been taken from Holboe \& Bourg. ${ }^{3}$ We further approximate the diffusion of interfacial water molecules and ions (i.e. those within 3 water layers $/ 45 \AA$ of a clay mineral surface, but not contributing to any crystalline hydrates) analogously to the diffusion of water in a 10 -water layer nanopore. ${ }^{3}$ All other water molecules and ions are assumed to diffuse with bulk like properties. 


\section{Results}

The following is a list of results which are referenced to in the main article:

- Figure 5: The evolution of system volume as a function of dry bulk density and waterto-clay mass ratio.

- Figure 6: The fraction of accessible pore space as a function of TPI probe radius calculated for the disordered clay system at various dry bulk densities.

- Figure 7: The solvent accessible surface area as a function of dry bulk density calculated from data on $P S D_{1}$.

- Figure 8: The fraction of pore space available to form 1-layer, 2-layer, and 3-layer hydrates estimated based on $P S D_{1}$.

- Figure 9: The anion-accessible porosity of compacted Na-smectite plotted as a function of ionic strength at different dry bulk densities.

- Figure 10: The evolution of enthalpy of the simulation of the disordered clay system (solid line) and the ideal models of $n$-layer hydrates (blue dots) as a function of $m_{w} / m_{c}$.

- Figure 11: The mean-squared displacement and consequent diffusion coefficients of water molecules in orthogonal directions calculated over various time displacements $\tau$

- Figure 12: The diffusion coefficients of sodium ions as a function of dry bulk density and water-to-clay mass ratio. Also presented is the the ratio of sodium ion to water molecule diffusion constants within the clay matrix at each point during dehydration. 


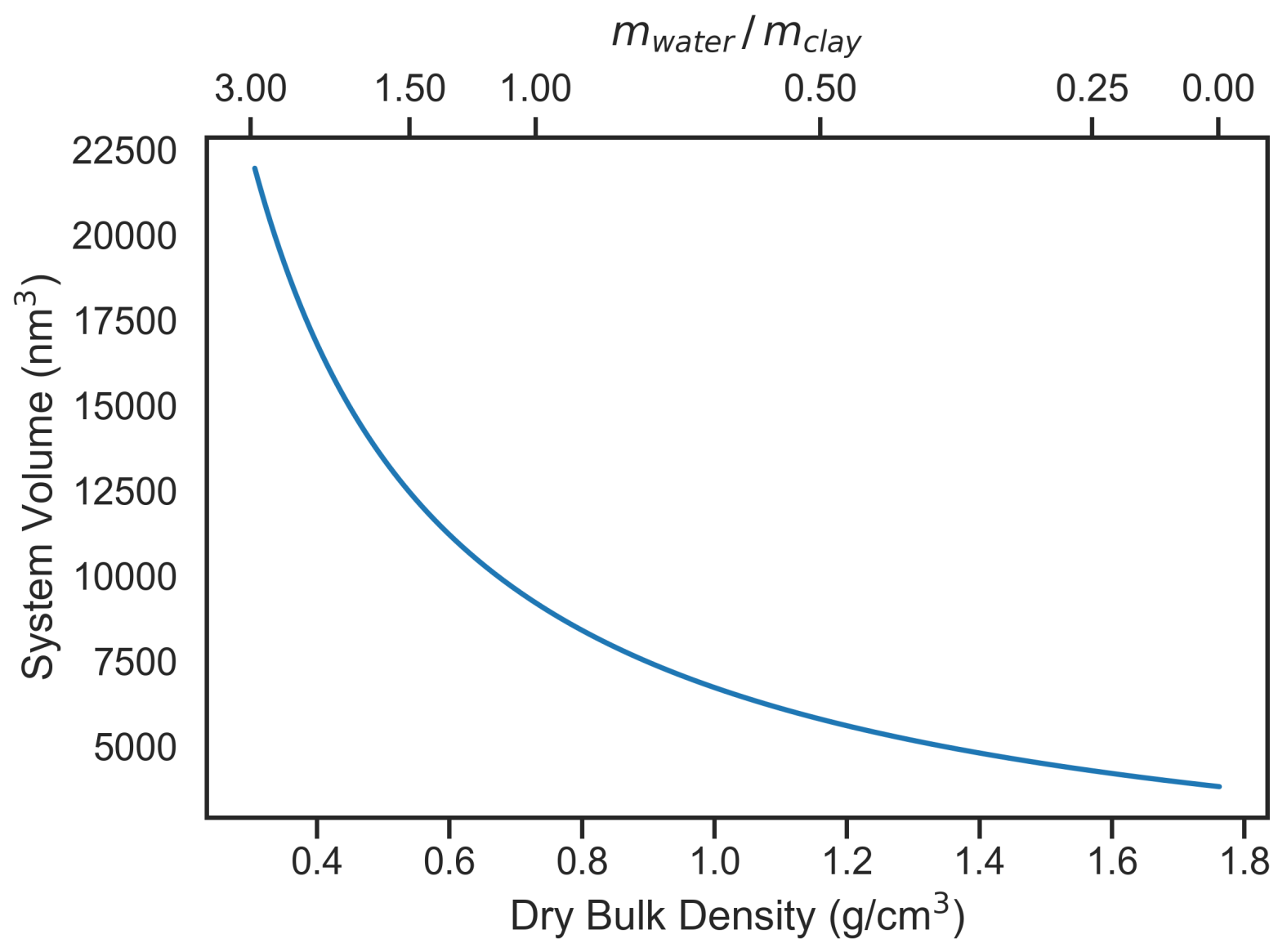

Figure 5: The evolution of system volume as a function of dry bulk density and water-to-clay mass ratio. 


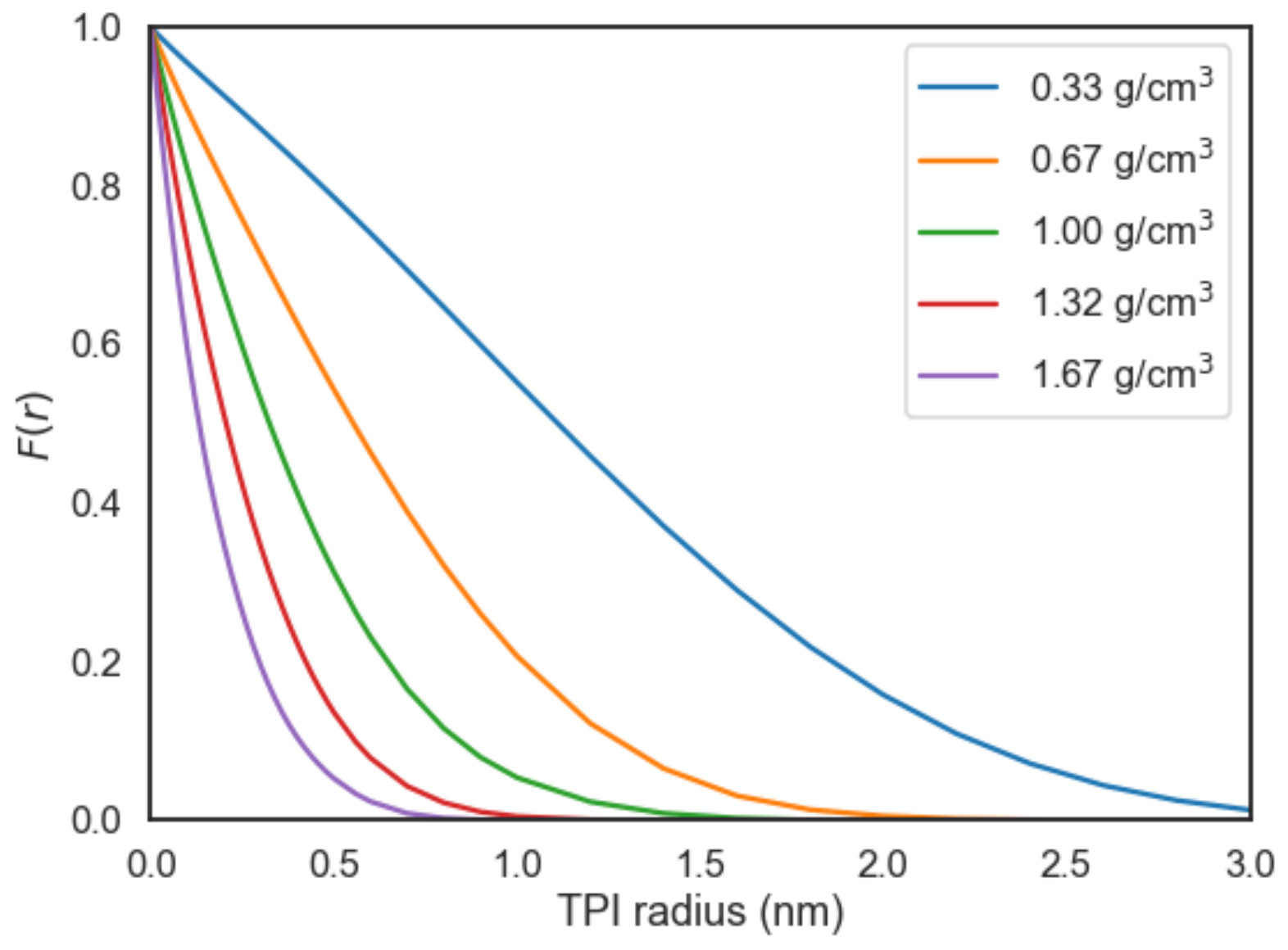

Figure 6: The fraction of accessible pore space as a function of TPI probe radius calculated for the disordered clay system at various dry bulk densities. 


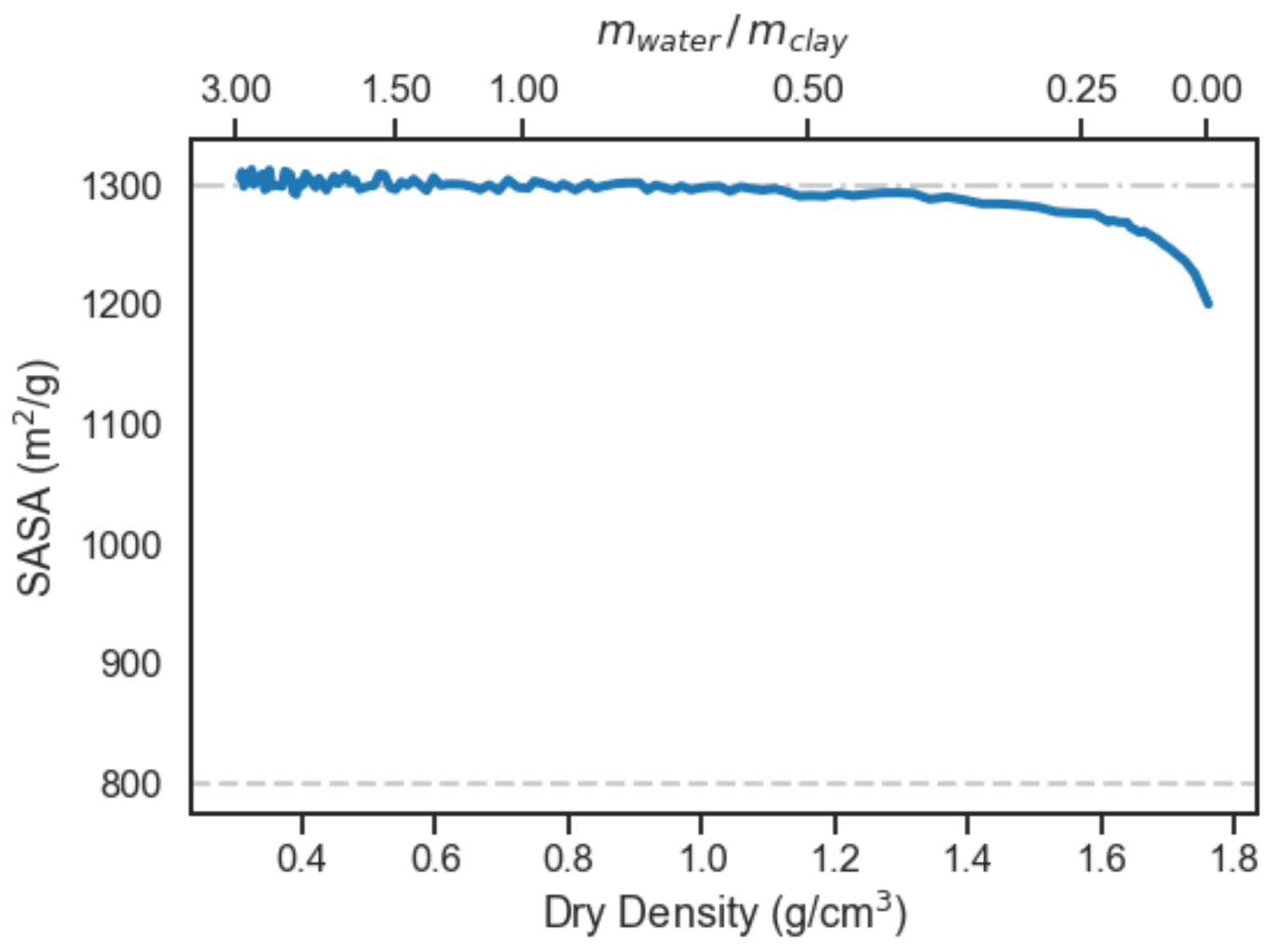

Figure 7: The solvent accessible surface area as a function of dry bulk density calculated from data on $P S D_{1}$. The horizonal dashed line shows the value expected based on the montmorillonite unit cell structure (for the specific surface area contributed by basal surfaces) and the dimensions of our hexagonal particles (for the specific surface area contributed by edge surfaces. The slight decrease in the SASA from $\approx 1300 \mathrm{~m}^{2} / \mathrm{g}$ to $\approx 1200 \mathrm{~m}^{2} / \mathrm{g}$ as dry bulk density increases above $1.4 \mathrm{~g} / \mathrm{cm}^{3}$ likely derives from the increasing face-to-face, edge-to-face and edge-to-edge steric interactions that occur as the clay matrix is compacted. 


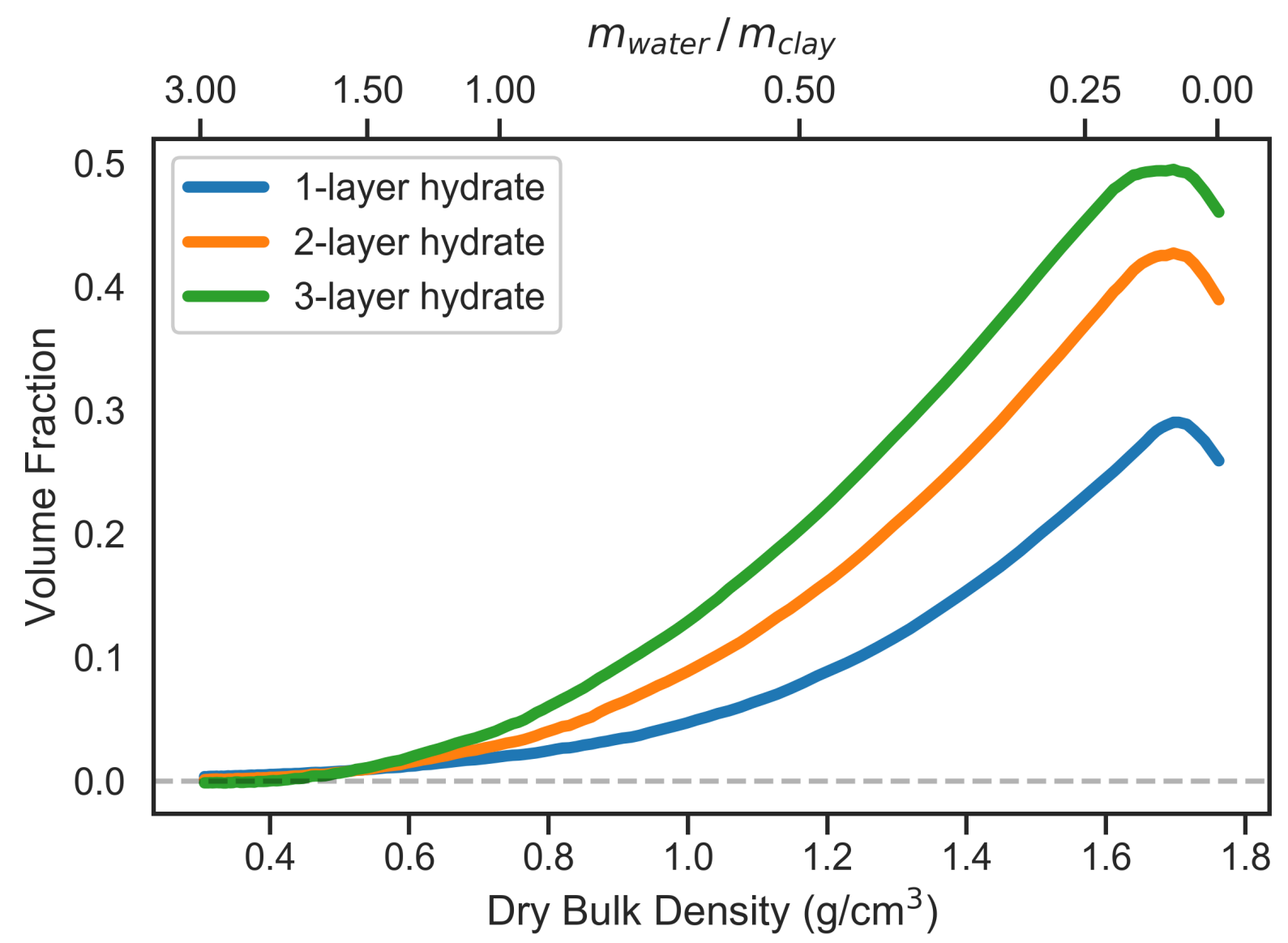

Figure 8: The fraction of pore space available to form 1-layer, 2-layer, and 3-layer hydrates estimated based on $P S D_{1}$. We observe a simultaneous, and approximately equal, increase in the pores available to form all types of crystalline hydrates up to a dry bulk density of $1.2 \mathrm{~g} / \mathrm{cm}^{3}$ and a water-to-clay mass ratio of approximately 0.50 . 


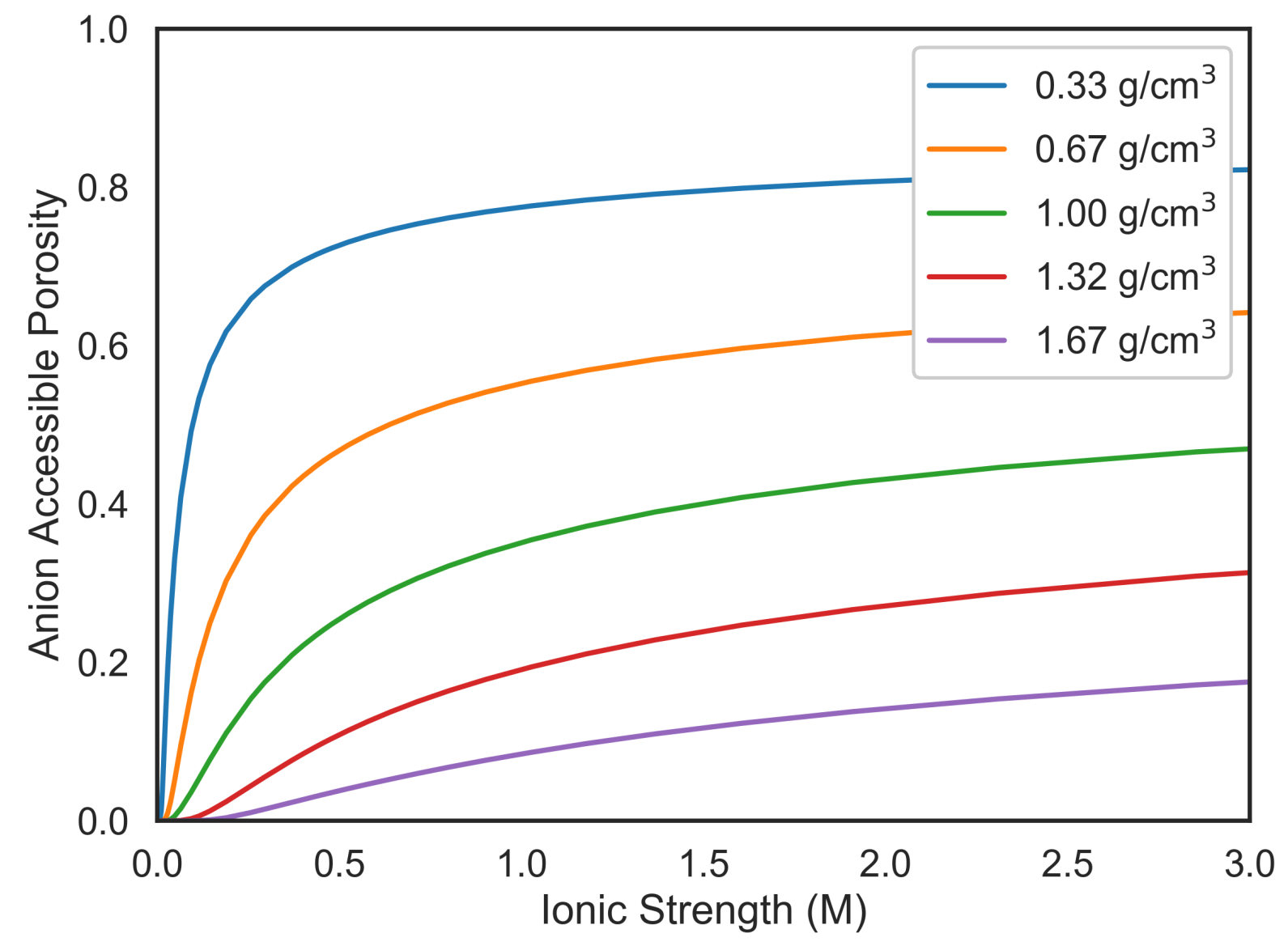

Figure 9: The anion-accessible porosity of compacted Na-smectite plotted as a function of ionic strength at different dry bulk densities. 


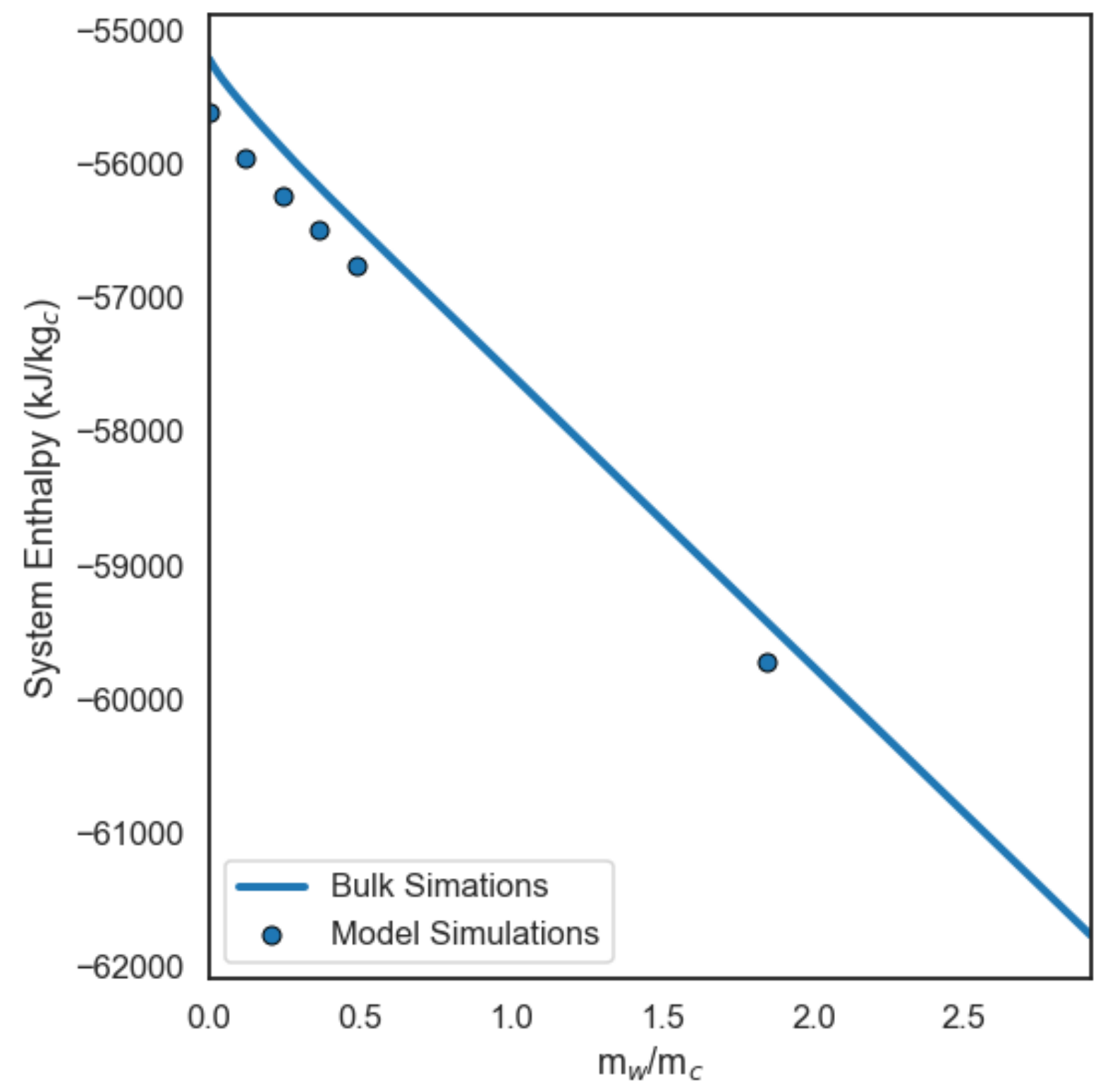

Figure 10: The evolution of enthalpy of the simulation of the disordered clay system (solid line) and the ideal models of $n$-layer hydrates (blue dots) as a function of $m_{w} / m_{c}$. Above a water-to-clay mass ratio of approximately 0.5 we observe a linear decrease in the system enthalpy as $m_{w} / m_{c}$ increases. The systematic difference between the MD simulations of the bulk system and those of the ideal periodic basal surfaces is due to the existence of clay edge surfaces in the bulk simulation that are not present in the models of ideal systems. 

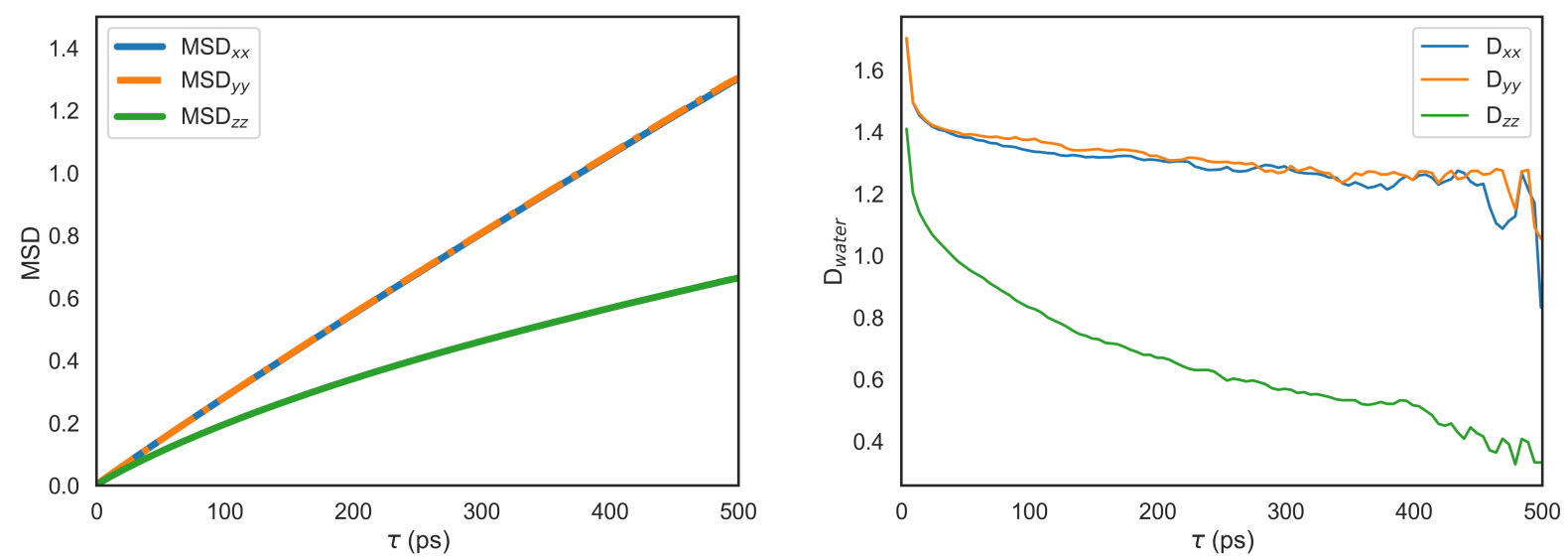

Figure 11: (Left) The mean-squared displacement of water molecules in orthogonal directions calculated over various time displacements $\tau$ for a dry bulk density of approximately 1.15 $\mathrm{g} / \mathrm{cm}^{3}$. (Right) The consequent diffusion coefficients of water molecules calculated at a dry bulk density of $1.15 \mathrm{~g} / \mathrm{cm}^{3}$ for various time displacements.
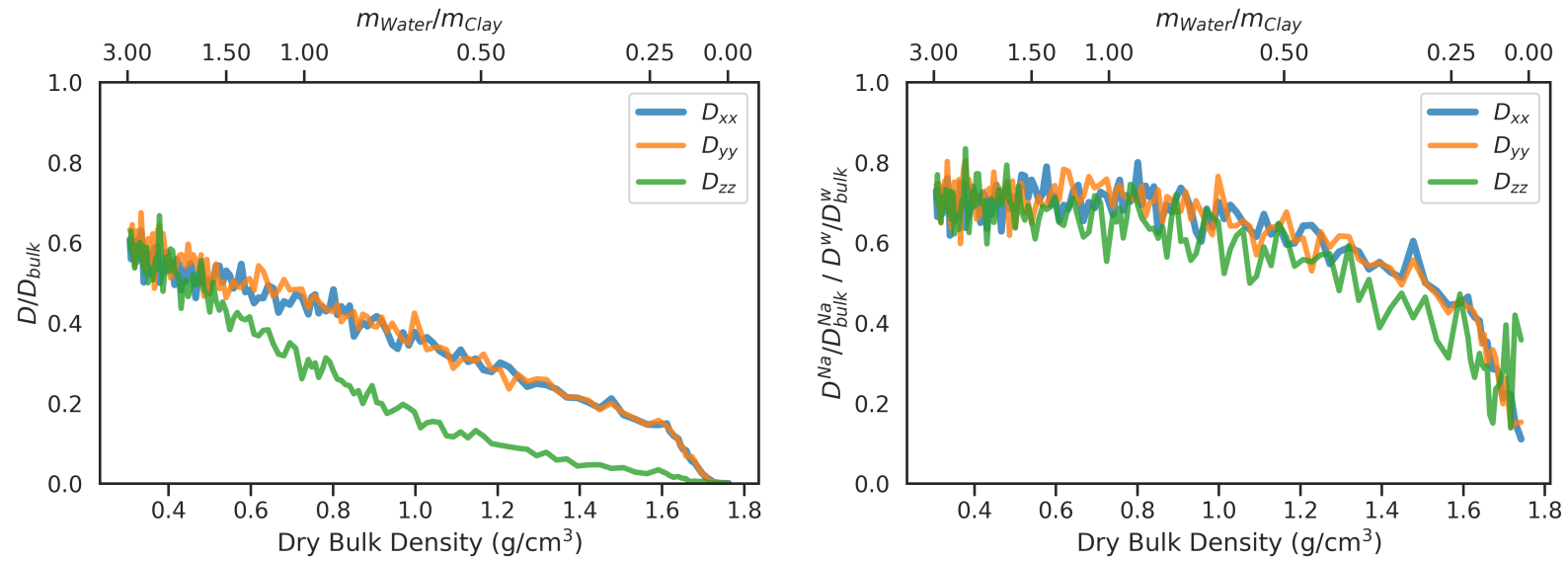

Figure 12: (Left) The diffusion coefficients of sodium ions as a function of dry bulk density and water-to-clay mass ratio. (Right) The ratio of sodium ion to water molecule diffusion constants within the clay matrix at each point during dehydration. 


\section{References}

(1) Lee, J. H.; Guggenheim, S. Single crystal X-ray refinement of pyrophyllite-1Tc. American Mineralogist 1981, 66, 350-357.

(2) Pauling, L. The principles determining the structure of complex ionic crystals. Journal of the american chemical society 1929, 51, 1010-1026.

(3) Holmboe, M.; Bourg, I. C. Molecular dynamics simulations of water and sodium diffusion in smectite interlayer nanopores as a function of pore size and temperature. The Journal of Physical Chemistry C 2014, 118, 1001-1013.

(4) Teich-McGoldrick, S. L.; Greathouse, J. A.; Jove-Colon, C. F.; Cygan, R. T. Swelling properties of montmorillonite and beidellite clay minerals from molecular simulation: comparison of temperature, interlayer cation, and charge location effects. The Journal of Physical Chemistry C 2015, 119, 20880-20891.

(5) White, G. N.; Zelazny, L. Analysis and Implications of the Edge Structure of Dioctahedral Phyllosilicates. CLAYS CLAY MINER. Clays Clay Miner 1988, 36, 141.

(6) Cygan, R. T.; Liang, J.-J.; Kalinichev, A. G. Molecular models of hydroxide, oxyhydroxide, and clay phases and the development of a general force field. The Journal of Physical Chemistry B 2004, 108, 1255-1266.

(7) Lammers, L. N.; Bourg, I. C.; Okumura, M.; Kolluri, K.; Sposito, G.; Machida, M. Molecular dynamics simulations of cesium adsorption on illite nanoparticles. Journal of colloid and interface science 2017, 490, 608-620.

(8) Liu, X.; Cheng, J.; Sprik, M.; Lu, X.; Wang, R. Interfacial structures and acidity of edge surfaces of ferruginous smectites. Geochimica et Cosmochimica Acta 2015, 168, $293-301$. 
(9) Ho, T. A.; Greathouse, J. A.; Wang, Y.; Criscenti, L. J. Atomistic Structure of Mineral Nano-aggregates from Simulated Compaction and Dewatering. Scientific reports $\mathbf{2 0 1 7}$ 7, 15286.

(10) Allen, M. P.; Tildesley, D. J. Computer simulation of liquids; Oxford university press, 1991.

(11) Abraham, M. J.; Murtola, T.; Schulz, R.; Páll, S.; Smith, J. C.; Hess, B.; Lindahl, E. GROMACS: High performance molecular simulations through multi-level parallelism from laptops to supercomputers. SoftwareX 2015, 1, 19-25.

(12) Bondi, A. van der Waals volumes and radii. The Journal of physical chemistry 1964, $68,441-451$.

(13) Muurinen, A.; Carlsson, T.; Root, A. Bentonite pore distribution based on SAXS, chloride exclusion and NMR studies. Clay Minerals 2013, 48, 251-266.

(14) Ferrage, E. Investigation of the interlayer organization of water and ions in smectite from the combined use of diffraction experiments and molecular simulations. A review of methodology, applications, and perspectives. Clays and Clay Minerals 2016, 64, $348-373$.

(15) Gelb, L. D.; Gubbins, K. Pore size distributions in porous glasses: a computer simulation study. Langmuir 1999, 15, 305-308.

(16) Sarkisov, L.; Harrison, A. Computational structure characterisation tools in application to ordered and disordered porous materials. Molecular Simulation 2011, 37, 1248-1257.

(17) Torquato, S.; Avellaneda, M. Diffusion and reaction in heterogeneous media: Pore size distribution, relaxation times, and mean survival time. The Journal of chemical physics 1991, 95, 6477-6489. 
(18) Daly, K. B.; Benziger, J. B.; Debenedetti, P. G.; Panagiotopoulos, A. Z. Massively parallel chemical potential calculation on graphics processing units. Computer Physics Communications 2012, 183, 2054-2062.

(19) Sato, H. Thermodynamic model on swelling of bentonite buffer and backfill materials. Physics and Chemistry of the Earth, Parts $A / B / C$ 2008, 33, S538-S543.

(20) Overbeek, J. T. G. On the electrostatic interaction in macroionic solutions and suspensions. The Journal of chemical physics 1987, 87, 4406-4408.

(21) Smalley, M. V. Clay swelling and colloid stability; CRC Press, 2006.

(22) Sposito, G. Thermodynamics of swelling clay-water systems. Soil Science 1972, 114, 243-249.

(23) Frenkel, D.; Smit, B. Understanding molecular simulation: from algorithms to applications; Elsevier, 2001; Vol. 1. 